\title{
BEST PROXIMITY PAIRS AND NASH EQUILIBRIUM PAIRS
}

\author{
WON KyU Kim and SANGHo Kum
}

\begin{abstract}
Main purpose of this paper is to combine the optimal form of Fan's best approximation theorem and Nash's equilibrium existence theorem into a single existence theorem simultaneously. For this, we first prove a general best proximity pair theorem which includes a number of known best proximity theorems. Next, we will introduce a new equilibrium concept for a generalized Nash game with normal form, and as applications, we will prove new existence theorems of Nash equilibrium pairs for generalized Nash games with normal form.
\end{abstract}

\section{Introduction}

In 1969, Fan proved the well-known best approximation theorem which generalizes the Tychonoff fixed point theorem as follows:

Theorem $\mathbf{A}([8])$. If $K$ is a non-empty compact convex subset of a locally convex Hausdorff topological vector space $E$ with a continuous seminorm $p$, and $f: K \rightarrow E$ is a single valued continuous function, then there exists an element $\bar{x} \in K$ such that

$$
p(f(\bar{x})-\bar{x})=d_{p}(f(\bar{x}), K):=\inf \{p(f(\bar{x})-y) \mid y \in K\} .
$$

Since then, a number of generalizations of this theorem have been obtained in various directions by several authors (e.g., see $[2,3,5,13,18-23])$. Indeed, Reich [18] has shown that even if $K$ is a non-empty approximately $p$-compact convex subset of a locally convex Hausdorff topological vector space $E$ with a relatively compact image $f(K)$, then the same conclusion holds. Later, Segal and Singh [21] have extended this result to convex valued continuous multifunctions. Even though a best approximation theorem guarantees the existence of an approximate solution, it is contemplated to find an approximate solution which is optimal. In this direction, Srinivasan and Veeramani [23] have proved the general forms of existence theorems for best proximity pairs, and Kim and Lee [12] prove two general existence theorems of best proximity pairs in a recent paper.

Received December 19, 2006; Revised December 12, 2007.

2000 Mathematics Subject Classification. Primary 47N10, 91A40.

Key words and phrases. best proximity pairs, Nash equilibrium pair. 
On the other hand, in 1951, Nash established the pioneering result on the existence of equilibrium for generalized games as follows:

Theorem B ([15]). Let I be a finite set of players. Assume that for all $i \in I$,

(a) the set $X_{i} \subset \mathbb{R}^{k_{i}}$ is non-empty compact and convex;

(b) the function $f_{i}: X:=\prod_{i \in I} X_{i} \rightarrow \mathbb{R}$ is continuous on $X$;

(c) the function $y_{i} \mapsto f_{i}\left(x_{1}, \ldots, x_{i-1}, y_{i}, x_{i+1}, \ldots, x_{n}\right)$ is concave on $X_{i}$.

Then there exists a Nash equilibrium $\bar{x}=\left(\bar{x}_{i}\right)_{i \in I} \in X$ such that for each $i \in I$,

$$
f_{i}\left(\bar{x}_{1}, \ldots, \bar{x}_{i}, \ldots, \bar{x}_{n}\right) \geq f_{i}\left(\bar{x}_{1}, \ldots, x_{i}, \ldots, \bar{x}_{n}\right) \text { for all } x_{i} \in X_{i} .
$$

Next, in 1977, Friedman [9] established a generalization of Theorem B using the quasi-concavity assumption on every payoff function. Since then, the classical results of Nash [14, 15], Debreu [4] and Friedman [9] have served as basic references for the existence of Nash equilibrium for non-cooperative generalized games. In all of them, convexity of strategy spaces, continuity and concavity/quasi-concavity of the payoff functions were assumed. Till now, there have been a number of generalizations, and also many applications of those theorems have been found in several areas, e.g., see [1, 9] and references therein.

In this paper, we will combine the optimal form of Theorem A and Theorem $\mathrm{B}$ into a single existence theorem simultaneously. For this purpose, we first prove a general best proximity pair theorem which generalizes the theorems due to Srinivasan and Veeramani [23, 24], Kim and Lee [12], and others. Next, we will introduce a new concept of Nash equilibrium pair in a generalized Nash game with normal form, and as applications of the best proximity pair theorem, we will prove new existence theorems of Nash equilibrium pairs for generalized Nash games with normal form which includes Theorem B and its generalizations due to Nikaido and Isoda [16], Kim and Lee [11], and others.

\section{Preliminaries}

We begin with some notations and definitions. Let $I$ be any (possibly infinite) index set. For each $i \in I$, let $X_{i}$ be a non-empty topological space and denote $X_{\hat{i}}:=\Pi_{j \in I \backslash\{i\}} X_{j}$, and $X:=\Pi_{i \in I} X_{i}=X_{i} \times X_{\hat{i}}$. If $x=$ $\left(x_{1}, \ldots, x_{n}, \ldots\right) \in X$, we shall write $x_{\hat{\imath}}=\left(x_{1}, \ldots, x_{i-1}, x_{i+1}, \ldots, x_{n}, \ldots\right) \in X_{\hat{i}}$, and if $x_{i} \in X_{i}$ and $x_{\hat{i}} \in X_{\hat{i}}$, we shall use the notation $x:=\left(x_{i}, x_{\hat{i}}\right)=$ $\left(x_{1}, \ldots, x_{i-1}, x_{i}, x_{i+1}, \ldots, x_{n}, \ldots\right) \in X$.

Let $I$ be a finite (or an infinite) index set. For each $i \in I$, let $X_{i}$ and $Y_{i}$ be non-empty subsets of a normed linear space $E$ with a norm $\|\cdot\|$, and the metric $d(x, y)$ for $E$ is induced by the norm, i.e., $d(x, y)=\|x-y\|$. Then we can use the following notations as in [12,24]: for each $i, j \in I$,

$$
\begin{aligned}
& d\left(X_{i}, Y_{j}\right):=\inf \left\{d(x, y) \mid x \in X_{i}, y \in Y_{j}\right\} \\
& X_{i}^{o}:=\left\{x \in X_{i} \mid \text { for each } j \in I, \exists y_{j} \in Y_{j} \text { such that } d\left(x, y_{j}\right)=d\left(X_{i}, Y_{j}\right)\right\} \\
& Y_{i}^{o}:=\left\{y \in Y_{i} \mid \text { for each } j \in I, \exists x_{j} \in X_{j} \text { such that } d\left(x_{j}, y\right)=d\left(X_{j}, Y_{i}\right)\right\}
\end{aligned}
$$


And we denote $X^{\circ}:=\Pi_{i \in I} X_{i}^{o}$ and $Y^{o}:=\Pi_{i \in I} Y_{i}^{\circ}$. If $X_{i}=\{x\}$, then $d\left(X_{i}, Y_{j}\right)$ is written as $d\left(x, Y_{j}\right)$. Also, if $X_{i}=\{x\}$ and $Y_{j}=\{y\}$, then $d(x, y)$ denotes $d\left(X_{i}, Y_{j}\right)$ which is precisely $\|x-y\|$.

If $X_{i}$ and $Y_{j}$ are non-empty compact and convex subsets of a normed linear space $E$, then when $|I|=1$, we can see that $X_{i}^{o}$ and $Y_{i}^{o}$ are both non-empty compact and convex; on the other hand, when $|I|>1$, it is easy to see that $X_{i}^{o}$ or $Y_{i}^{o}$ may be an empty set in general. In fact, when $E=\mathbb{R}^{2}$ with the Euclidean metric $d$ and $X_{1}=X_{2}=\{x \in E \mid d(x,(0,0)) \leq 1\}$;

$$
Y_{1}=\{x \in E \mid d(x,(3,0)) \leq 1\}, \quad Y_{2}=\{x \in E \mid d(x,(0,3)) \leq 1\}
$$

then each $Y_{i}^{o}$ is non-empty compact and convex but $X_{i}^{o}$ is an empty set.

For each $i \in I$, let $X_{i}$ and $Y_{i}$ be two non-empty subsets of a normed linear space $E$, and let $T_{i}: X=\Pi_{i \in I} X_{i} \rightarrow 2^{Y_{i}}$ be a multifunction. Then the pair $\left(\bar{x}_{i}, T_{i}(\bar{x})\right) \in X_{i} \times Y_{i}$ is called the best proximity pair for $T_{i}$ if $d\left(\bar{x}_{i}, T_{i}(\bar{x})\right)=$ $d\left(X_{i}, Y_{i}\right)$ for each $i \in I$. Then the best proximity pair theorem seeks an appropriate solution $\bar{x} \in X$ which is optimal. In fact, the best proximity pair theorem analyzes the conditions which the problem of minimizing the realvalued function $x \mapsto d\left(x_{i}, T_{i}(x)\right)$ has a simultaneous solution for each $i \in I$. In the best proximity pair, whenever $X_{i}=Y_{i}$ for each $i \in I$, then $\bar{x}$ is actually the fixed point for the multifunction $T=\Pi_{i \in I} T_{i}$, i.e., $\bar{x} \in T(\bar{x})$. Still there have been a number of generalized theorems on the existence of best proximity pairs which can be regarded as the optimal forms of Theorem A by several authors, e.g., see $[2,12,19,20,23]$.

Let $X$ be a non-empty subset of a normed linear space $E$, and consider the metric projection map $P_{X}: E \rightarrow 2^{X}$ defined by for each $z \in E$,

$$
P_{X}(z):=\{x \in X \mid\|z-x\|=d(z, x)=d(z, X)\} .
$$

Then, it is well-known that if $X$ is non-empty compact and convex, then $P_{X}(x)$ is a non-empty compact and convex subset of $X$, and the multifunction $P_{X}$ is upper semicontinuous in $X$. For the properties of the metric projection, see [18-20].

Next, we recall the following definition due to Kim and Lee which generalizes the concavity condition:

Definition 1 ([11]). Let $X$ be a non-empty topological space, $Y$ an arbitrary set. Then $f: X \times Y \rightarrow \mathbb{R}$ is called $\mathcal{C}$-concave on $X$ if for every $n \geq 2$, whenever $n$ points $x_{1}, \ldots, x_{n} \in X$ are arbitrarily given, there exists a continuous function $\phi_{n}:[0,1]^{n} \rightarrow X$ such that

$$
f\left(\phi_{n}\left(\lambda_{1}, \ldots, \lambda_{n}\right), y\right) \geq \lambda_{1} f\left(x_{1}, y\right)+\cdots+\lambda_{n} f\left(x_{n}, y\right)
$$

for all $\lambda_{i} \in[0,1], i=1, \ldots, n$, with $\sum_{i=1}^{n} \lambda_{i}=1$, and for all $y \in Y$.

Remark. As remarked in [11], the concavity clearly implies the $\mathcal{C}$-concavity by letting $\phi_{n}\left(\lambda_{1}, \ldots, \lambda_{n}\right):=\lambda_{1} x_{1}+\cdots+\lambda_{n} x_{n}$, whenever $x_{1}, \ldots, x_{n} \in X$ are given; however, we do not know the implications between the quasi-concavity and the $\mathcal{C}$-concavity. 
Also recall that a topological space $X$ is said to have the fixed point property (or is a fixed point space) [5] if every continuous mapping $f: X \rightarrow X$ has a fixed point in $X$. Clearly this property is topologically invariant, and note that the product of two fixed point spaces need not be a fixed point space. In contrast with finite products, an infinite product of non-empty compact fixed point spaces will be a fixed point space whenever every finite product of those spaces is a fixed point space, e.g. see [5, p.174].

A multifunction $T: X \rightarrow 2^{Y}$ from a topological space $X$ to a Hausdorff topological vector space $Y$ is said to be a Kakutani multifunction [13] if the following conditions are satisfied: (i) $T$ is upper semicontinuous; (ii) either $T(x)$ is singleton for each $x \in X$ or for each $x \in X, T(x)$ is a non-empty compact convex subset of $Y$. A multifunction $S: X \rightarrow 2^{Y}$ is said to be a Kakutani factorizable multifunction [13] if it can be expressed as a composition of finitely many Kakutani multifunctions, i.e., $S=T_{1} \circ T_{2} \circ \cdots \circ T_{n}$, and each $T_{i}$ is a Kakutani factorizable multifunction.

\section{Existence of best proximity pairs}

The following Lassonde fixed point theorem for Kakutani factorizable multifunctions is a generalization of the well-known Fan-Glicksberg's fixed point theorem, and it is essential in proving the existence of best proximity pairs:

Theorem 1 ([13]). If $X$ is a non-empty compact and convex subset of a locally convex Hausdorff topological vector space, then any Kakutani factorizable multifunction $T: X \rightarrow 2^{X}$ has a fixed point, i.e., there exists a point $\bar{x} \in X$ such that $\bar{x} \in T(\bar{x})$.

Now we will prove a new existence theorem for the best proximity pairs as follow:

Theorem 2. Let $I$ be an (possibly infinite) index set and for each $i \in I$, let $X_{i}$ and $Y_{i}$ be non-empty compact and convex subsets of a normed linear space $E$, and let $A_{i}: X=\Pi_{i \in I} X_{i} \rightarrow 2^{Y_{i}}$ be an upper semicontinuous multifunction in $X$ such that $A_{i}(x)$ is a non-empty closed and convex subset of $Y_{i}$ for each $x \in X$. Assume that for each $i \in I$,

$$
A_{i}(x) \cap Y_{i}^{o} \neq \emptyset \quad \text { for all } x \in X .
$$

Then there exists a point $\bar{x}=\left(\bar{x}_{i}\right)_{i \in I} \in X$ of best proximity pairs such that for each $i \in I, d\left(\bar{x}_{i}, A_{i}(\bar{x})\right)=d\left(X_{i}, Y_{i}\right)$.

Proof. For each $i \in I$, since $X_{i}$ and $Y_{i}$ are non-empty compact and convex, it is easy to prove that $Y_{i}^{o}$ is a non-empty compact and convex subset of $Y_{i}$. In fact, by the assumption (*), $Y_{i}^{o}$ is non-empty. Let $i \in I$ be arbitrarily fixed. In order to show $Y_{i}^{o}$ is convex, let $y_{1}, y_{2} \in Y_{i}^{o}$ be given. Then, for each $j \in I$, there exist $x_{j}^{1}, x_{j}^{2} \in X_{j}$ such that $d\left(x_{j}^{k}, y_{k}\right)=d\left(X_{j}, Y_{i}\right)$ for $k=1,2$. For any $\lambda \in(0,1)$, we let $\hat{y}:=\lambda y_{1}+(1-\lambda) y_{2}$, and $\hat{x}_{j}:=\lambda x_{j}^{1}+(1-\lambda) x_{j}^{2}$. Since $X_{j}$ 
and $Y_{i}$ are convex, $\hat{x}_{j} \in X_{j}$ and $\hat{y} \in Y_{i}$. Then for each $j \in I$, we have

$$
\begin{aligned}
d\left(\hat{x}_{j}, \hat{y}\right) & =\left\|\left(\lambda x_{j}^{1}+(1-\lambda) x_{j}^{2}\right)-\left(\lambda y_{1}+(1-\lambda) y_{2}\right)\right\| \\
& =\left\|\lambda\left(x_{j}^{1}-y_{1}\right)+(1-\lambda)\left(x_{j}^{2}-y_{2}\right)\right\| \\
& \leq \lambda\left\|x_{j}^{1}-y_{1}\right\|+(1-\lambda)\left\|x_{j}^{2}-y_{2}\right\|=d\left(X_{j}, Y_{i}\right)
\end{aligned}
$$

so that $\hat{y} \in Y_{i}^{\circ}$, and hence $Y_{i}^{\circ}$ is convex. Similarly, the convexity for $X_{i}^{\circ}$ can be proved. Next, in order to show that $Y_{i}^{\circ}$ is a closed subset of $Y_{i}$, let $\left(y_{n}\right)_{n \in \mathbb{N}}$ be a sequence in $Y_{i}^{\circ}$ which converges to $\tilde{y} \in Y_{i}$. For each $j \in I$, let $k_{j}:=d\left(X_{j}, Y_{i}\right)$. Then, since $y_{n} \in Y_{i}^{o}$ for each $n \in \mathbb{N}$, there exists $x_{j}^{n} \in X_{j}$ such that $\left\|x_{j}^{n}-y_{n}\right\|=k_{j}$ for each $j \in I$. Since $\left\{x_{j}^{n}\right\} \subset X_{j}$ and $X_{j}$ is compact, there exists a convergent subsequence $\left(x_{j}^{n_{k}}\right)$ of $\left(x_{j}^{n}\right)$ which converges to $\tilde{x}_{j} \in X_{j}$.

Then we can see that

$$
\begin{aligned}
k_{j} \leq\left\|\tilde{x}_{j}-\tilde{y}\right\| & =\left\|\tilde{x}_{j}-x_{j}^{n_{k}}+x_{j}^{n_{k}}-y_{n_{k}}+y_{n_{k}}-\tilde{y}\right\| \\
& \leq\left\|\tilde{x}_{j}-x_{j}^{n_{k}}\right\|+\left\|x_{j}^{n_{k}}-y_{n_{k}}\right\|+\left\|y_{n_{k}}-\tilde{y}\right\| .
\end{aligned}
$$

Since $\left\|\tilde{x}_{j}-x_{j}^{n_{k}}\right\| \rightarrow 0$ and $\left\|y_{n_{k}}-\tilde{y}\right\| \rightarrow 0$, and $\left\|x_{j}^{n_{k}}-y_{n_{k}}\right\|=k_{j}$, we have $\left\|\tilde{x}_{j}-\tilde{y}\right\|=k_{j}$ so that $\tilde{y} \in Y_{i}^{\circ}$. Therefore, $Y_{i}^{\circ}$ is a closed subset of $Y_{i}$ so that $Y^{\circ}:=\prod_{i \in I} Y_{i}^{o}$ is a non-empty closed and convex subset of $Y=\prod_{i \in I} Y_{i}$.

Also, it is known that for each $i \in I$, the metric projection map $P_{X_{i}}: E \rightarrow$ $2^{X_{i}}$ is upper semicontinuous in $E$ such that $P_{X_{i}}(z)$ is a non-empty compact and convex subset of $X_{i}$ for each $z \in E$ (e.g., see [18, 24]).

For each $i \in I$, we now define a multifunction $A_{i}^{\prime}: X \rightarrow 2^{Y_{i}^{\circ}}$ by

$$
A_{i}^{\prime}(x):=A_{i}(x) \cap Y_{i}^{\circ} \quad \text { for each } x \in X=\prod_{i \in I} X_{i} .
$$

Then, by the assumptions, each $A_{i}^{\prime}(x)$ is non-empty compact and convex in $Y_{i}^{0}$, and $A_{i}^{\prime}$ is upper semicontinuous in $X$.

Finally, we introduce two multifunctions $A^{\prime}: X \rightarrow 2^{Y^{\circ}}$, defined by

$$
A^{\prime}(x):=\Pi_{i \in I} A_{i}^{\prime}(x) \quad \text { for each } x=\left(x_{i}\right)_{i \in I} \in \Pi_{i \in I} X_{i} ;
$$

and $P_{X}^{\prime}: \Pi_{i \in I} Y_{i} \rightarrow 2^{\Pi_{i \in I} X_{i}}$, defined by

$$
P_{X}^{\prime}(y):=\Pi_{i \in I} P_{X_{i}}\left(y_{i}\right) \quad \text { for each } y=\left(y_{i}\right)_{i \in I} \in \Pi_{i \in I} Y_{i} .
$$

Since $A_{i}^{\prime}$ and $P_{X_{i}}$ are upper semicontinuous with compact and convex values for each $i \in I$, by Lemma 3 in [6], $A^{\prime}$ and $P_{X}^{\prime}$ are both upper semicontinuous such that each $A^{\prime}(x)$ is non-empty compact and convex in $Y^{o}$, and each $P_{X}^{\prime}(y)$ is non-empty compact and convex in $X$. Hence, $A^{\prime}$ and $P_{X}^{\prime}$ are Kakutani multifunctions so that the composition map $P_{X}^{\prime} \circ A^{\prime}: X \rightarrow 2^{X}$ is a Kakutani factorizable multifunction. Therefore, by Theorem 1, there exists a fixed point $\bar{x}=\left(\bar{x}_{i}\right)_{i \in I} \in X$ such that $\bar{x} \in\left(P_{X}^{\prime} \circ A^{\prime}\right)(\bar{x})$. Then, $\left(\bar{x}_{i}\right)_{i \in I} \in P_{X}^{\prime}\left(A^{\prime}\left(\left(\bar{x}_{i}\right)_{i \in I}\right)\right)$ so that there exists an $\left(\bar{y}_{i}\right)_{i \in I} \in \Pi_{i \in I} Y_{i}^{o}$ such that $\left(\bar{y}_{i}\right)_{i \in I} \in A^{\prime}(\bar{x})=\Pi_{i \in I}\left(A_{i}(\bar{x}) \cap Y_{i}^{o}\right)$ and $\bar{x}_{i} \in P_{X_{i}}\left(\bar{y}_{i}\right)$ for each $i \in I$. Since each $\bar{y}_{i}$ is an element in $Y_{i}^{o}$, there exists an $x_{i}^{\prime} \in X_{i}$ such that $d\left(x_{i}^{\prime}, \bar{y}_{i}\right)=d\left(X_{i}, Y_{i}\right)$ 
for each $i \in I$. Therefore, for each $i \in I$, we have

$$
d\left(\bar{x}_{i}, A_{i}(\bar{x})\right) \leq d\left(\bar{x}_{i}, \bar{y}_{i}\right)=d\left(X_{i}, \bar{y}_{i}\right) \leq d\left(x_{i}^{\prime}, \bar{y}_{i}\right)=d\left(X_{i}, Y_{i}\right)
$$

so that $d\left(\bar{x}_{i}, A_{i}(\bar{x})\right)=d\left(X_{i}, Y_{i}\right)$ which completes the proof.

Remarks. (i) The assumptions of Theorem 2 is different from those of Theorem 1 and 2 in [12], and the conclusions have slight different forms. However, Theorem 2 can be regarded as an infinite generalization of Theorem 1 and 2 in $[12]$.

(ii) If $X_{i}=Y_{j}$ and $A_{i}: X \rightarrow 2^{X_{i}}$ for each $i, j \in I$, then the assumption that " $A_{i}(x) \cap Y_{i}^{o} \neq \emptyset "$ is automatically satisfied. In fact, $Y_{i}^{o}=X_{i}$. Similarly, in case $Y_{i}=E$ for each $i \in I$, the assumption that " $A_{i}(x) \cap Y_{i}^{o} \neq \emptyset$ " is automatically satisfied and $Y_{i}^{o}=E$. In those cases, the conclusion means that $\bar{x}$ is a fixed point for $\Pi_{i \in I} A_{i}$, which implies the Bohnenblust-Karlin fixed point theorem [5].

(iii) When $|I|=1$, Theorem 2 generalizes Theorem 3.4 of SrinivasanVeeramani [24] by relaxing the condition " $A\left(X^{\circ}\right) \subseteq Y^{\circ}$ " into the weaker condition " $A(x) \cap Y^{o} \neq \emptyset$ " for each $x \in X$.

Next we give a simple example which is suitable for Theorem 2, but the previous theorems due to Srinivasan-Veeramani [23, 24], Kim-Lee [12] and others can not be applied:

Example 1. Let $E=\mathbb{R}^{2}$ with the Euclidean metric $d$, and for each $1=1,2$, let

$$
\begin{aligned}
X_{i} & :=\{(x, 0) \mid 0 \leq x \leq 2\} \\
Y_{i} & :=\{(x, y) \mid 0 \leq x \leq 2,1 \leq y \leq 2\} .
\end{aligned}
$$

And let $X:=X_{1} \times X_{2}$ and $Y:=Y_{1} \times Y_{2}$. Then it is easy to see that $X_{i}^{\circ}=X_{i}$ and $Y_{i}^{o}=\{(y, 1) \mid 0 \leq y \leq 2\}$, and $d\left(X_{i}, Y_{i}\right)=1$ for each $i=1,2$. For each $i=1,2$, let $A_{i}: X \rightarrow 2^{Y_{i}}$ be a multifunction defined by for each $\left(\left(x_{1}, 0\right),\left(x_{2}, 0\right)\right) \in X$,

$$
\begin{aligned}
& A_{1}\left(\left(x_{1}, 0\right),\left(x_{2}, 0\right)\right):=\left\{\left(2-x_{1}, y\right)|1 \leq y \leq| x_{1}-1 \mid+1\right\} \\
& A_{2}\left(\left(x_{1}, 0\right),\left(x_{2}, 0\right)\right):=\left\{\left(x_{2}, y\right)|1 \leq y \leq| x_{2}-1 \mid+1\right\} .
\end{aligned}
$$

Then, it is clear that each $A_{i}\left(\left(x_{1}, 0\right),\left(x_{2}, 0\right)\right)$ is a non-empty closed and convex subset of $Y_{i}$. Also, it is easy to see that $A_{i}$ is upper semicontinuous in $X$. Then, for each $\left(\left(x_{1}, 0\right),\left(x_{2}, 0\right)\right) \in X^{\circ}=X$, it is easy to see that $A_{1}\left(\left(x_{1}, 0\right),\left(x_{2}, 0\right)\right) \cap$ $Y_{1}^{o}=\left\{\left(2-x_{1}, 1\right)\right\} \neq \emptyset$ and $A_{2}\left(\left(x_{1}, 0\right),\left(x_{2}, 0\right)\right) \cap Y_{2}^{o}=\left\{\left(x_{2}, 1\right)\right\} \neq \emptyset$; however, $A_{i}\left(\left(x_{1}, 0\right),\left(x_{2}, 0\right)\right) \nsubseteq Y_{i}^{o}$ for some $\left(\left(x_{1}, 0\right),\left(x_{2}, 0\right)\right) \in X$, and $X=X_{1} \times X_{2}$ is a product set so that the theorems due to Srinivasan-Veeramani [23, 24], KimLee [12] can not be applied. However, all the hypotheses of Theorem 2 are satisfied so that there exists a point $\bar{x}=((1,0),(1,0)) \in X$ of best proximity pairs such that $d\left((1,0), A_{i}(\bar{x})\right)=d\left(X_{i}, Y_{i}\right)=1$ for each $i=1,2$. 


\section{Existence of Nash equilibrium pairs}

We first recall the equilibrium concept in the game theory due to Nash [15]. Let $I=\{1, \ldots, n\}$ be a set of players. A non-cooperative Nash game (or $n$-person game) $\Gamma=\left(X_{i} ; f_{i}\right)_{i \in I}$ of normal form is an ordered $2 n$-tuple $\left(X_{1}, \ldots, X_{n} ; f_{1}, \ldots, f_{n}\right)$, where for each player $i \in I$, the non-empty set $X_{i}$ is the player's pure strategy space, and $f_{i}: X=\prod_{i=1}^{n} X_{i} \rightarrow \mathbb{R}$ is the player's payoff function. The set $X$, joint strategy space, is the Cartesian product of the individual strategy sets, and an element of $X_{i}$ is called a strategy. A strategy $n$-tuple $\left(\bar{x}_{1}, \ldots, \bar{x}_{n}\right) \in X$ is called a Nash equilibrium for $\Gamma$ if the following system of inequalities holds: for each $i=1, \ldots, n$,

$$
f_{i}\left(\bar{x}_{i}, \bar{x}_{\hat{i}}\right) \geq f_{i}\left(x_{i}, \bar{x}_{i}\right) \text { for all } x_{i} \in X_{i} .
$$

This pioneering concept on the existence of equilibrium for a non-cooperative $n$-person game was established by Nash in 1951, and is regraded as the basic result in equilibrium theories. Here it should be noted that it is possible to generalize that the set of players $I$ may be infinite in the model of a game.

In recent papers [23, 24], Srinivasan and Veeramani considered a general economic situation which is motivated to introduce the equilibrium pair concept in a constrained generalized games as follow. Suppose that goods are manufactured and sold in different locations. Each location (or player) can be a manufacturing as well as selling unit. It is agreed that the ultimate place where the goods get sold would be determining the payoff for the goods. Let there be $n$ such locations. For each location, two strategies $X_{i}$ and $Y_{i}$ are associated, one to that of manufacturing unit and other to that of selling unit. Knowing the manufacturing strategy $x_{\hat{i}} \in X_{\hat{i}}:=\Pi_{j \in I \backslash\{i\}} X_{j}$ of all other locations, the choice of selling strategy at the $i$ th location is restricted to $A_{i}(x) \subseteq Y_{i}$. Also, let the payoff function $f_{i}: Y=\Pi_{i=1}^{n} Y_{i} \rightarrow \mathbb{R}$ at the $i$ th location be given such that $f_{i}$ is the real-valued function at the given strategy $y \in Y$ which can maximize the payoff associated with the $i$ th location. In fact, the preferred strategies are strongly dependent to the consumer's behavior at the $i$ th location. Under those settings, they investigated the system of multifunctions $A_{i}: X \rightarrow 2^{Y_{i}}$ and $f_{i}: Y \rightarrow \mathbb{R}$ for each $i \in I$. Moreover, the cost involved in the transportation of goods to different places should also be taken into account. In fact, the transporting cost from $x_{i}$ to $A_{i}(x)$ can be considered as $d\left(x_{i}, A_{i}(x)\right)$, and so it is very reasonable that its payoff function $f_{i}\left(y_{i}, y_{\hat{i}}\right)$ on $A_{i}(x)$ can have a maximum whenever $d\left(x_{i}, A_{i}(x)\right)$ has a minimum value $d\left(X_{i}, Y_{i}\right)$. In these situations, one can not expect an equilibrium for the Nash game of normal form by applying the previous results in $[3,4,9,11,14-17]$ because the strategy sets $X_{i}$ and $Y_{i}$ may be different in general.

In $[23,24]$, as applications of their best proximity theorems, Srinivasan and Veeramani proved existence theorems of equilibrium pair for constrained generalized games by using the conditions (A) and (B), respectively. And, as remarked, the condition (c) of Definition 4.3 in [23] has less economic information 
for constrained generalized games. This is the motivation of our equilibrium pair concept for a generalized Nash games, and also we shall prove an existence theorem of equilibrium pair for constrained generalized games without assuming the conditions (A) in [24] nor (B) in [23].

In this section, as an application of our best proximity pair theorem (Theorem 2), we shall prove an existence theorem of equilibrium pair for a generalized Nash games. Now we will introduce a new concept of equilibrium pairs in generalized Nash games as follows:

Definition 2. Let $I$ be a finite or an infinite set of players or locations. For each $i \in I$, let $X_{i}$ be a non-empty set of manufacturing commodities, and $Y_{i}$ be a non-empty set of selling commodities. A non-cooperative generalized Nash game $\Gamma=\left(X_{i}, Y_{i} ; A_{i}, f_{i}\right)_{i \in I}$ of normal form is defined as a family of ordered quadruples $\left(X_{i}, Y_{i} ; A_{i}, f_{i}\right)$ where $X_{i}$ and $Y_{i}$ are non-empty subsets of a normed linear space $E$, and $A_{i}: X=\Pi_{j \in I} X_{j} \rightarrow 2^{Y_{i}}$ is a constraint correspondence, and $f_{i}: Y=\Pi_{i=1}^{n} Y_{i} \rightarrow \mathbb{R}$ is the player's payoff function. A Nash equilibrium pair for $\Gamma$ is a pair of points $(\bar{x}, \bar{y})=\left(\left(\bar{x}_{i}\right)_{i \in I},\left(\bar{y}_{i}\right)_{i \in I}\right) \in X \times Y$ such that for each $i \in I$,

$$
\begin{gathered}
\bar{y}_{i} \in A_{i}(\bar{x}) \quad \text { with } d\left(\bar{x}_{i}, \bar{y}_{i}\right)=d\left(\bar{x}_{i}, A_{i}(\bar{x})\right)=d\left(X_{i}, Y_{i}\right), \text { and } \\
f_{i}\left(\bar{y}_{i}, \bar{y}_{\hat{i}}\right) \geq f_{i}\left(y_{i}, \bar{y}_{\hat{i}}\right) \text { for all } y_{i} \in A_{i}\left(x_{i}\right) .
\end{gathered}
$$

In particular, when $I=\{1, \ldots, n\}$, we may call $\Gamma$ a generalized n-person game of normal form.

Remarks. (i) The transporting cost conditions $\left\|x-y_{i}\right\|=d\left(X, \hat{Y}_{i}\right)$ in Theorem 4.5 in [23] and $\|x-y\|=d(X, Y)$ in Definition 4.3 in [24] have reasonable economic meanings in their settings, and it should be noted that our condition is related with the Definition 4.3 in [24] as

$$
\|x-y\|=d(X, Y) \Longleftrightarrow\left\|x_{i}-y_{i}\right\|=d\left(X_{i}, Y_{i}\right) \text { for each } i \in I .
$$

(ii) When $X_{i}=Y_{i}$ and $A_{i}(x)=X_{i}$ for each $i \in I$ and $x \in X$ in Definition 2, then the Nash equilibrium pair can be reduced to the standard definitions of equilibrium in the game theory due to Nash [14, 15], Debreu [4] or Friedman [9], and so the Nash game $\Gamma^{\prime}=\left(X_{i}, ; f_{i}\right)_{i \in I}$ can be considered as a subgame of the generalized Nash game $\Gamma=\left(X_{i}, Y_{i} ; A_{i}, f_{i}\right)_{i \in I}$ of normal form. For more economic meanings and interpretations, see [3,9, 14-17].

In [17], in order to obtain a Nash equilibrium for a game $\Gamma$, Nikaido and Isoda define the total sum of payoff functions $H: X \times X \rightarrow \mathbb{R}$ associated with the non-cooperative game $\Gamma=\left(X_{i}, ; f_{i}\right)_{i \in I}$, as follows:

$$
H(x, y):=\sum_{i=1}^{n} f_{i}\left(y_{1}, \ldots, y_{i-1}, x_{i}, y_{i+1}, \ldots, y_{n}\right)
$$

for every $x=\left(x_{1}, \ldots, x_{n}\right), y=\left(y_{1}, \ldots, y_{n}\right) \in X=\prod_{i=1}^{n} X_{i}$.

Here we note that if for each $i \in I$, the function $y_{i} \mapsto f_{i}\left(y_{i}, x_{\hat{i}}\right)$ is concave as in Theorem B, then the function $x \mapsto H(x, y)$ is concave; and so it is $\mathcal{C}$-concave. 
As mentioned before, we can generalize the total sum of payoff functions for the infinite generalized Nash game $\Gamma$. For this, we shall need the following. Let $I$ be an infinite set of players, and let $\Gamma=\left(X_{i}, Y_{i} ; A_{i}, f_{i}\right)_{i \in I}$ be a noncooperative generalized Nash game of normal form. For each $i \in I, f_{i}: Y_{i} \times$ $Y_{\hat{i}} \rightarrow \mathbb{R}$ is said to be unconditionally summable if for each $z_{i} \in Y_{\dot{i}}$ and $y_{\hat{i}} \in Y_{\hat{i}}$, any rearrangement $\sum_{j \in I} f_{j}\left(z_{j}, y_{j}\right)$ of the infinite sum $\sum_{i \in I} f_{i}\left(z_{i}, y_{\hat{i}}\right)$ converges to the same real value. Note that if $\Gamma=\left(X_{i} ; f_{i}\right)_{i \in I}$ is a finite Nash game, then each $f_{i}$ is automatically unconditionally summable.

Using the unconditionally summablity, let us define the total sum of payoff functions $H: Y \times Y \rightarrow \mathbb{R}$ associated with the infinite generalized Nash game $\Gamma=\left(X_{i}, Y_{i} ; A_{i}, f_{i}\right)_{i \in I}$ as follows:

$$
H(z, y):=\sum_{i \in I} f_{i}\left(z_{i}, y_{i}\right) \text { for each } y=\left(y_{i}\right)_{i \in I}, z=\left(z_{i}\right)_{i \in I} \in Y .
$$

The following is essential in proving the existence of generalized Nash equilibrium:

Lemma 1. Let $I$ be an infinite set of players and let $\Gamma=\left(X_{i} ; f_{i}\right)_{i \in I}$ be a noncooperative Nash game of normal form where $f_{i}$ is unconditionally summable for each $i \in I$. If there exists a point $\bar{x} \in X=\prod_{i \in I} X_{i}$ with $H(\bar{x}, \bar{x}) \geq H(x, \bar{x})$ for any $x \in X$, then $\bar{x}$ is a Nash equilibrium for $\Gamma$.

Proof. For each $i \in I$ and for any $x=\left(x_{i}, \bar{x}_{i}\right) \in X, x_{i} \in X_{i}$, by substitution, we can see that

$$
f_{i}\left(\bar{x}_{i}, \bar{x}_{\hat{i}}\right) \geq f_{i}\left(x_{i}, \bar{x}_{\hat{i}}\right) \text { for all } x_{i} \in X_{i}
$$

so that $\bar{x}$ is a Nash equilibrium.

We shall need the following which is an infinite generalization of Theorem 1 in [11] where their proof can be worked without any changes:

Lemma 2 ([11]). Let $I$ be an infinite set of players, and let $\Gamma=\left(X_{i} ; f_{i}\right)_{i \in I}$ be a non-cooperative Nash game of normal form. For each $i \in I, f_{i}: X_{i} \times X_{\hat{i}} \rightarrow \mathbb{R}$ is unconditionally summable and satisfies the following:

(i) the set $X=X_{i} \times X_{\hat{i}}$ is non-empty compact and has the fixed-point property;

(ii) the function $H(x, y)$ is continuous on $X \times X$;

(iii) the function $x \mapsto H(x, y)$ is $\mathcal{C}$-concave on $X$.

Then $\Gamma$ has at least one Nash equilibrium.

Remark. As remarked in [11], Lemma 2 generalizes the previous equilibrium existence theorems due to Nash [17] and Nikaido and Isoda [16] in several aspects.

As an application of Theorem 2, we will prove the existence of Nash equilibrium pairs for a generalized Nash game with infinite set of players as follows: 
Theorem 3. Let $I$ be an infinite set of players and let $\Gamma=\left(X_{i}, Y_{i} ; A_{i}, f_{i}\right)_{i \in I}$ be a generalized Nash game of normal form. For each $i \in I$, the payoff function $f_{i}: Y=Y_{i} \times Y_{\hat{i}} \rightarrow \mathbb{R}$ is unconditionally summable and assume the following conditions:

(1) $X_{i}$ and $Y_{i}$ are non-empty compact and convex subsets of a normed linear space $E$;

(2) $A_{i}: X=\prod_{j \in I} X_{j} \rightarrow 2^{Y_{i}}$ is an upper semicontinuous correspondence such that each $A_{i}(x)$ is a non-empty closed and convex subset of $Y_{i}$, and satisfies the condition $(*)$ in Theorem 2 ;

(3) the payoff function $f_{i}: Y_{i} \times Y_{\hat{i}} \rightarrow \mathbb{R}$ is continuous;

(4) the function $y_{i} \mapsto f_{i}\left(y_{i}, x_{\hat{i}}\right)$ is concave.

Then there exists an equilibrium pair $(\bar{x}, \bar{y})=\left(\left(\bar{x}_{i}\right)_{i \in I},\left(\bar{y}_{i}\right)_{i \in I}\right) \in X \times Y$ for $\Gamma$, i.e., for each $i \in I, \quad \bar{y}_{i} \in A_{i}(\bar{x})$ such that $d\left(\bar{x}_{i}, A_{i}(\bar{x})\right)=d\left(X_{i}, Y_{i}\right)$ and

$$
f_{i}\left(\bar{y}_{i}, \bar{y}_{\hat{i}}\right) \geq f_{i}\left(y_{i}, \bar{y}_{\hat{i}}\right) \text { for all } y_{i} \in A_{i}(\bar{x}) \text {. }
$$

Proof. For each $i \in I$, since $A_{i}$ satisfies the whole assumptions of Theorem 2, there exists a point $\bar{x}=\left(\bar{x}_{i}\right)_{i \in I} \in X$ of best proximity pairs, i.e., for each $i \in I$, $\left\{\bar{x}_{i}\right\} \times A_{i}(\bar{x}) \subseteq X_{i} \times Y_{i}$ such that $d\left(\bar{x}_{i}, A_{i}(\bar{x})\right)=d\left(X_{i}, Y_{i}\right)$. It remains to show the existence of Nash equilibrium on the constraint set $\Pi_{i \in I} A_{i}(\bar{x})$. Since $A_{i}(\bar{x})$ is a non-empty closed convex subset of $Y_{i}$, we can now restrict the generalized Nash game $\Gamma=\left(X_{i}, Y_{i} ; A_{i}, f_{i}\right)_{i \in I}$ to the Nash subgame $\Gamma^{\prime}=\left(A_{i}(\bar{x}) ; f_{i}\right)_{i \in I}$, and also restrict the domain of the total sum of payoff functions $H$ on $\mathcal{A} \times \mathcal{A}$ where $\mathcal{A}:=\Pi_{i \in I} A_{i}(\bar{x})$. Then the whole assumptions of Lemma 2 are satisfied so that there exists a Nash equilibrium $\left(\bar{y}_{i}\right)_{i \in I} \in \Pi_{i \in I} A_{i}(\bar{x})$ for the Nash subgame $\Gamma^{\prime}=\left(A_{i}(\bar{x}) ; f_{i}\right)_{i \in I}$, i.e., for each $i \in I$,

$$
f_{i}\left(\bar{y}_{i}, \bar{y}_{\hat{i}}\right) \geq f_{i}\left(y_{i}, \bar{y}_{\hat{i}}\right) \text { for all } y_{i} \in A_{i}(\bar{x}) .
$$

Therefore, we can obtain an equilibrium pair $(\bar{x}, \bar{y})=\left(\left(\bar{x}_{i}\right)_{i \in I},\left(\bar{y}_{i}\right)_{i \in I}\right) \in X \times$ $\mathcal{A} \subseteq X \times Y$ for the generalized Nash game $\Gamma$. This completes the proof.

Remark. In Theorem 3, we can relax the assumptions (3) and (4) into the following weaker assumptions without affecting the conclusion:

$\left(3^{\prime}\right)$ the total sum of payoff functions $H: Y \times Y \rightarrow \mathbb{R}$, defined by

$$
H(z, y):=\sum_{i \in I} f_{i}\left(z_{i}, y_{i}\right) \quad \text { for each } y=\left(y_{i}\right)_{i \in I},\left(z_{i}\right)_{i \in I} \in Y,
$$

satisfies the following conditions

(i) the function $(z, y) \mapsto H(z, y)$ is continuous on $Y \times Y$;

(ii) the function $z \mapsto H(z, y)$ is $\mathcal{C}$-concave on $Y$.

In Theorem 3, when $X_{i}=Y_{i}$ and $A_{i}(x)=X_{i}$ for each $i \in I$ and for all $x \in X$ where each $X_{i}$ is assumed to be contained in the Euclidean space $\mathbb{R}^{n}$, we can obtain the infinite generalization of the existence of Nash equilibrium (i.e., Theorem B): 
Corollary 1. Let $I$ be an infinite set of players and $\Gamma=\left(X_{i} ; f_{i}\right)_{i \in I}$ be a noncooperative Nash game where each $f_{i}$ is unconditionally summable. Assume that for each $i \in I$,

(a) the set $X_{i} \subset \mathbb{R}^{k_{i}}$ is non-empty compact and convex;

(b) the function $f_{i}$ is continuous;

(c) the function $y_{i} \mapsto f_{i}\left(y_{i}, x_{i}\right)$ is concave.

Then there exists at least one Nash equilibrium for $\Gamma$.

Here we note that the relations between the constraint multifunction $A_{i}$ and the payoff function $f_{i}$ on $A_{i}(x)$. In real market economies, whenever $i$ th player can minimize his/her transporting cost from $x_{i}$ to $A_{i}(x)$, it is very natural that its payoff function $f_{i}\left(y_{i}, y_{i}\right)$ can be maximizable. In fact, if $y_{i} \in A_{i}(x)$ with $d\left(x_{i}, y_{i}\right)>d\left(x_{i}, A_{i}(x)\right)=d\left(X_{i}, Y_{i}\right)$, then it is plausible that $\left.f_{i}\right|_{A_{i}(x)}$ can not attain a maximum at $y_{i}$. Therefore, the following compatibility assumption on the relation between $A_{i}$ and $f_{i}$ is reasonable in the generalized Nash game with normal form: for each $i \in I$,

$(\dagger)$

If $\left.f_{i}\right|_{A_{i}(x)}$ attains a maximum at $y_{i}$ in $A_{i}(x)$, then $d\left(x_{i}, y_{i}\right)=d\left(x_{i}, A_{i}(x)\right)$.

Adding the above assumption $(\dagger)$ in Theorem 3, we can obtain the existence of Nash equilibrium pairs for a generalized Nash game $\Gamma$ as follows:

Theorem 4. Let $I$ be an infinite set of players and let $\Gamma=\left(X_{i}, Y_{i} ; A_{i}, f_{i}\right)_{i \in I}$ be a generalized Nash game with normal form. For each $i \in I$, the payoff function $f_{i}: Y_{i} \times Y_{\hat{i}} \rightarrow \mathbb{R}$ is unconditionally summable and assume the following conditions:

(1) $X_{i}$ and $Y_{i}$ are non-empty compact and convex subsets of a normed linear space $E$;

(2) $A_{i}: X=\Pi_{j \in I} X_{j} \rightarrow 2^{Y_{i}}$ is an upper semicontinuous correspondence such that each $A_{i}(x)$ is a non-empty closed and convex subset of $Y_{i}$, and satisfies the condition $(*)$ in Theorem 2 and the condition $(\dagger)$;

(3) the total sum of payoff functions $H: Y \times Y \rightarrow \mathbb{R}$ satisfies the following conditions

(i) the function $(z, y) \mapsto H(z, y)$ is continuous on $Y \times Y$;

(ii) the function $z \mapsto H(z, y)$ is $\mathcal{C}$-concave on $Y$.

Then there exists a Nash equilibrium pair $(\bar{x}, \bar{y})=\left(\left(\bar{x}_{i}\right)_{i \in I},\left(\bar{y}_{i}\right)_{i \in I}\right) \in X \times Y$ for $\Gamma$, i.e., for each $i \in I, \bar{y}_{i} \in A_{i}(\bar{x})$ such that $d\left(\bar{x}_{i}, \bar{y}_{i}\right)=d\left(\bar{x}_{i}, A_{i}(\bar{x})\right)=d\left(X_{i}, Y_{i}\right)$ and

$$
f_{i}\left(\bar{y}_{i}, \bar{y}_{\hat{i}}\right) \geq f_{i}\left(y_{i}, \bar{y}_{\hat{i}}\right) \text { for all } y_{i} \in A_{i}(\bar{x}) \text {. }
$$

Proof. By the condition ( $\dagger$ ), we can obtain that the equilibrium pair $(\bar{x}, \bar{y}) \in$ $X \times Y$ in the conclusion of Theorem 3 is actually the Nash equilibrium pair for $\Gamma$.

Remark. As already mentioned, the condition $(\dagger)$ can be reformulated as the contrapositive form: 
(†) If $d\left(x_{i}, y_{i}\right)>d\left(x_{i}, A_{i}(x)\right)$ for some $y_{i} \in A_{i}(x)$, then $\left.f_{i}\right|_{A_{i}(x)}$ can not attain a maximum at $y_{i}$ in $A_{i}(x)$.

Finally, we shall give an example of a generalized 2-person game which is suitable for Theorem 4 , but the previous equilibrium existence theorems in $[14-17,23,24]$ can not be applied:

Example 2. Let $\Gamma=\left(X_{i}, Y_{i} ; A_{i}, f_{i}\right)_{i=1,2}$ be a generalized 2-person game such that $E=\mathbb{R}^{2}$ with the Euclidean metric $d$, and for each $i=1,2$,

$$
\begin{aligned}
X_{i} & :=\{(x, 0) \mid 0 \leq x \leq 2\} ; \\
Y_{i} & :=\{(x, y) \mid 0 \leq x \leq 2,1 \leq y \leq 3\} .
\end{aligned}
$$

And let $X:=X_{1} \times X_{2}$ and $Y:=Y_{1} \times Y_{2}$. Then it is easy to see that $X_{i}^{o}=X_{i}$ and $Y_{i}^{o}=\{(y, 1) \mid 0 \leq y \leq 2\}$, and $d\left(X_{i}, Y_{i}\right)=1$ for each $i=1,2$.

Let $A_{i}: X \rightarrow 2^{Y_{i}}$ and $f_{i}: Y \rightarrow \mathbb{R}$ be correspondences defined by for each $x=\left(\left(x_{1}, 0\right),\left(x_{2}, 0\right)\right) \in X$ and $\left(\left(y_{1}, y_{2}\right),\left(z_{1}, z_{2}\right)\right) \in Y$,

$$
\begin{aligned}
A_{1}\left(\left(x_{1}, 0\right),\left(x_{2}, 0\right)\right) & :=\left\{\left(x_{1}, a\right) \mid 1 \leq a \leq\left(x_{1}-1\right)^{2}+2\right\} ; \\
A_{2}\left(\left(x_{1}, 0\right),\left(x_{2}, 0\right)\right) & :=\left\{\left(2-x_{2}, b\right) \mid 1 \leq b \leq\left(x_{1}-1\right)^{2}+2\right\} ; \\
f_{1}\left(\left(y_{1}, y_{2}\right),\left(z_{1}, z_{2}\right)\right) & :=1-y_{2}^{2} ; \quad f_{2}\left(\left(y_{1}, y_{2}\right),\left(z_{1}, z_{2}\right)\right):=1-z_{2}^{2} .
\end{aligned}
$$

Then, it is clear that each $A_{i}(x)$ is non-empty closed and convex subset of $Y_{i}$ for each $x \in X$ and $i=1,2$. Also, it is easy to see that $A_{i}$ is upper semicontinuous in $X$. And, we can see that $X^{\circ}=X, Y^{o}=\left\{\left(\left(y_{1}, 1\right),\left(y_{2}, 1\right)\right) \mid 0 \leq y_{1}, y_{2} \leq 3\right\}$, and $d\left(X_{i}, Y_{i}\right)=1$ for each $i=1,2$. Then, for each $x \in X^{o}=X$, it is easy to see that $A_{1}(x) \cap Y_{1}^{o}=\left\{\left(x_{1}, 1\right)\right\} \neq \emptyset$ and $A_{2}(x) \cap Y_{2}^{o}=\left\{\left(2-x_{2}, 1\right)\right\} \neq \emptyset$; however, $A_{i}((1,0),(1,0)) \nsubseteq Y_{i}^{\circ}$ for each $i=1,2$, and $A_{i}^{-1}(a, 1)$ is not open in $X$ for each $(a, 1) \in Y$ so that the previous theorems due to Debreu [4], Nash [14, 15], Kim-Lee [11, 12], Srinivasan-Veeramani [24] can not be applied in this model. It is easy to see that since each $f_{i}$ is continuous and concave, the assumptions (3)(i) and (ii) of Theorem 4 are satisfied so that there exists a Nash equilibrium pair $(\bar{x}, \bar{y}) \in X \times Y$ for $\Gamma$, that is, we can obtain that $\bar{x}=((1,0),(1,0)), \bar{y}=((1,1),(1,1))$ is the desired Nash equilibrium pair for the generalized 2-person game $\Gamma$. In fact, for each $i=1,2$, we have

$$
\begin{aligned}
\bar{y}_{i} & =(1,1) \in A_{i}((1,0),(1,0))=\{(1, a) \mid 1 \leq a \leq 2\} \\
1 & =d\left(\bar{x}_{i}, A_{i}(\bar{x})\right)=d\left(X_{i}, Y_{i}\right) ; \text { and } \\
0 & =f_{1}((1,1),(1,1)) \geq f_{1}\left(\left(y_{1}, y_{2}\right),(1,1)\right)=1-y_{2}^{2}, \quad \forall\left(y_{1}, y_{2}\right) \in A_{1}((1,0),(1,0)) ; \\
0 & =f_{2}((1,1),(1,1)) \geq f_{2}\left((1,1),\left(z_{1}, z_{2}\right)\right)=1-z_{2}^{2}, \quad \forall\left(z_{1}, z_{2}\right) \in A_{2}((1,0),(1,0)) .
\end{aligned}
$$

Acknowledgements. The authors would like to thank the referee for helpful comments.

\section{References}

[1] M. Avriel, W. E. Diewert, S. Schaible, and I. Zang, Generalized Concavity, Plenum Press, New York, 1988. 
[2] G. Beer and D. Pai, Proximal maps, prox maps and coincidence points, Numer. Funct. Anal. Optim. 11 (1990), no. 5-6, 429-448.

[3] K. C. Border, Fixed Point Theorems with Applications to Economics and Game Theory, Cambridge University Press, Cambridge, 1985.

[4] G. Debreu, A social equilibrium existence theorem, Proc. Nat. Acad. Sci. U. S. A. 38 (1952), 886-893.

[5] J. Dugundji and A. Granas, Fixed Point Theory. I, Polish Sci. Publ., Warsaw, 1982.

[6] K. Fan, Fixed-point and minimax theorems in locally convex topological linear spaces, Proc. Nat. Acad. Sci. U. S. A. 38 (1952), 121-126.

[7] - Minimax theorems, Proc. Nat. Acad. Sci. U. S. A. 39 (1953), 42-47.

[8] Extensions of two fixed point theorems of F. E. Browder, Math. Z. 112 (1969), $234-240$.

[9] J. Friedman, Oligopoly and the Theory of Games, North-Holland, Amsterdam, 1977.

[10] I. Joó, Answer to a problem of M. Horváth and A. Sövegjártó, Ann. Univ. Sci. Budapest. Eötvös Sect. Math. 29 (1986), 203-207 (1987).

[11] W. K. Kim and K. H. Lee, The existence of Nash equilibrium in n-person games with $\mathcal{C}$-concavity, Comput. Math. Appl. 44 (2002), no. 8-9, 1219-1228.

[12] Existence of best proximity pairs and equilibrium pairs, J. Math. Anal. Appl. 316 (2006), no. 2, 433-446.

[13] M. Lassonde, Fixed points for Kakutani factorizable multifunctions, J. Math. Anal. Appl. 152 (1990), no. 1, 46-60.

[14] J. Nash, Equilibrium points in n-person games, Proc. Nat. Acad. Sci. U. S. A. 36 (1950), 48-49.

[15] _ Non-cooperative games, Ann. of Math. (2) 54 (1951), 286-295.

[16] H. Nikaidô and K. Isoda, Note on non-cooperative convex games, Pacific J. Math. 5 (1955), 807-815.

[17] K. Nishimura and J. Friedman, Existence of Nash equilibrium in n-person games without quasiconcavity, Internat. Econom. Rev. 22 (1981), no. 3, 637-648.

[18] S. Reich, Approximate selections, best approximations, fixed points, and invariant sets, J. Math. Anal. Appl. 62 (1978), no. 1, 104-113.

[19] S. Sadiq Basha and P. Veeramani, Best proximity pairs and best approximations, Acta Sci. Math. (Szeged) 63 (1997), no. 1-2, 289-300.

[20] — Best proximity pair theorems for multifunctions with open fibres, J. Approx. Theory 103 (2000), no. 1, 119-129.

[21] V. M. Sehgal and S. P. Singh, A generalization to multifunctions of Fan's best approximation theorem, Proc. Amer. Math. Soc. 102 (1988), no. 3, 534-537.

[22] I. Singer, Best Approximation in Normed Linear Spaces by Elements of Linear Subspaces, Springer-Verlag, New York-Berlin, 1970.

[23] P. S. Srinivasan and P. Veeramani, On best proximity pair theorems and fixed-point theorems, Abstr. Appl. Anal. 2003 (2003), no. 1, 33-47.

[24] __ On existence of equilibrium pair for constrained generalized games, Fixed Point Theory Appl. 2004 (2004), no. 1, 21-29.

WON KYU Kim

DePaRTMENT OF Mathematics EdUCATION

Chungbuk National University

Cheonguu 361-763, Korea

E-mail address: wkkim@chungbuk.ac.kr 
SANGHO KUM

Department of Mathematics Education

ChUNGBUK NATIONAL UNIVERSTTY

Cheonguu 361-763, Korea

E-mail address: shkum@chungbuk.ac.kr 